\title{
Myocardial Strain Imaging in Asian Competitive Athletes - A Single Centre Study
}

\author{
Yann Shan Keh ${ }^{1,3}$, Puay Joo Tan ${ }^{1}$, Siang Chew Chai ${ }^{1}$, Benedict Tan $^{2}$, Khim Leng Tong ${ }^{1}$ * \\ ${ }^{1}$ Department of Cardiology, Changi General Hospital, Singapore \\ ${ }^{2}$ Department of Sports Medicine, Changi General Hospital, Singapore \\ ${ }^{3}$ Department Cardiology, National Heart Centre, Singapore
}

Email address:

tong.khim.leng@singhealth.com.sg (K. L. Tong)

${ }^{*}$ Corresponding author

\section{To cite this article:}

Yann Shan Keh, Puay Joo Tan, Siang Chew Chai, Benedict Tan, Khim Leng Tong. Myocardial Strain Imaging in Asian Competitive Athletes - A Single Centre Study. American Journal of Sports Science. Vol. 8, No. 1, 2020, pp. 17-21. doi: 10.11648/j.ajss.20200801.13

Received: January 24, 2020; Accepted: March 9, 2020; Published: March 23, 2020

\begin{abstract}
To date there has been limited literature pertaining to Athlete's Heart Syndrome in Asian athletes, especially for those in South East Asia. We performed a single center cross-sectional case-control study of elite athletes and controls, using current speckle-tracking echocardiography (STE) and tissue Doppler imaging (TDI)-based techniques. We reanalyzed previous data to further characterize the biomechanical changes in exercise induced cardiac remodeling elite athletes and controls at a tertiary hospital in Singapore. The Left Ventricular (LV) strain of the athletes' group was significantly lower as compared to the control group $(-19.0 \pm 2.0$ vs $-20.3 \pm 1.8, \mathrm{p}=0.011)$. Furthermore, both $\mathrm{LV}$ torsion $\left(14.3 \pm 17.8^{\circ} / \mathrm{s}\right.$ vs $\left.14.4 \pm 6.7^{\circ} / \mathrm{s}, \mathrm{p}=0.031\right)$ and Lateral S' (7.21 \pm 1.4 vs $8.7 \pm 1.6, \mathrm{p}=0.001)$ showed small but statistically significant decreases in the athletes' group versus the controls. The athletes group demonstrated a significantly lower Basal Right Ventricular (RV) free wall strain as compared to the controls $(-19.8 \pm 5.5$ vs $-26.5 \pm 6.4 \mathrm{P}<0.001)$. The mid RV strain was marginally higher in the athletes' group versus the controls $(-25.0 \pm 4.3$ vs $-24.7 \pm 15.3 \mathrm{P}=0.023)$. Our findings of impaired LV strain and torsion as well basal RV free walls strain in the South East Asian athletes group mirror studies done in Western cohorts. The higher mid RV free wall strain could represent compensatory response to the impaired basal RV function in athletes.
\end{abstract}

Keywords: Speckle-Tracking Echocardiography, Tissue Doppler Imaging, Athlete's Heart Syndrome, Exercise Induced Cardiac Remodeling, Asian Athletes

\section{Introduction}

Exercise induced cardiac remodeling or 'Athlete's Heart Syndrome' is defined as the various cardiac morphologic and functional adaptations which have been described in the hearts of the athletes. This has been traditionally been characterized on 2D echocardiography as a combination of morphologic changes such as ventricular enlargement, myocardial hypertrophy and atrial dilatation [1]. The recent advent of myocardial deformation techniques including Tissue Doppler Imaging (TDI), Strain Rate by 2D and 3D echocardiography have added greater clarity to the functional adaptations of Athlete's Heart Syndrome. [2] These techniques have also helped in differentiating exercise induced cardiac remodeling from other pathological conditions such as Hypertrophic Cardiomyopathy (HCM) and Arrhythmogenic Right Ventricle Cardiomyopathy (ARVC) [2].

Pulsed tissue Doppler imaging (TDI)-derived early diastolic myocardial velocity (e') of basal septal and basal lateral wall has been reported by other centers to be significantly higher in athletes as compared to sedentary controls. [3, 4] A e' peak velocity of $<11.5 \mathrm{~cm} / \mathrm{s}$ has been suggested as a marker to differentiate between athletes heart from HCM. [5]

Using 2D strain echocardiography, radial and transverse Left Ventricular (LV) strains were significantly higher in professional soccer players compared with controls, whereas longitudinal strain was lower. [6] There have been a number of adaptive changes in RV function characterized by strain imaging over the past 10 years, including an acute decrease 
in RV strain rate after intense endurance exercise and chronic adaptations such as mildly impaired RV global systolic function and significantly impaired basal RV free wall deformation on strain imaging. [6]

We recently published the results of trial performed in our center comparing basic echocardiographic parameters in between athletes and controls. [7] The athletes group demonstrated significantly larger LA LV and RV dimensions as compared to the controls group, with no difference in markers of diastology (E/A, E/E', IVRT and DT). Using current speckle-tracking echocardiography (STE) and tissue Doppler imaging (TDI)-based techniques, we reanalyzed previous data to further characterize the biomechanical changes in exercise induced cardiac remodeling.

\section{Methodology}

We performed a single center cross-sectional case-control study looking at the echocardiographic dimensions of elite athletes and controls at Changi General Hospital, a tertiary hospital in Singapore. All the athletes were recruited from various sports centers in Singapore while the control group consisted of age, gender and race matched healthy sedentary adults. Athletes were defined as individuals currently training in strength or endurance sports more than 3 times a week and $>40 \mathrm{~min}$ per session and competing at club level or above for $>1 \mathrm{yr}$. The subjects were excluded if they were either $<$ 21 years old, had limited echocardiographic windows, known hypertension, significant cardiac history or cardiac surgery. During post processing of the images, subjects whose images were deemed suboptimal during analysis were excluded from the study. The study was approved by the institutional review committee and complied with the principles of the Declaration of Helsinki.

\subsection{Study Design}

The study protocol has previously been described in our previous publication. [7] Images from the original studied were post processed using Echopac version 113, version 1.2 (GE Healthcare).

For global LV longitudinal strain (GLS) and LA strain, 2D images from the 3 apical views were captured for the longitudinal LA and LV myocardial strain. Images were standardized at 3 cardiac cycles with the frame rate of 60-80 Hz. All patients were in sinus rhythm. Speckle Tracking (STE) software was used to analyze 2D strain frame by frame throughout LV systole and during the LA reservoir phase. Segments that failed to track were excluded. Peak GLS were determined in the $18 \mathrm{LV}$ segments and peak atrial longitudinal strain of the 18 LA segments was determined during the reservoir phase. RV strain was analyzed using only the basal, mid and apical RV free wall.

2D images from the basal and apical parasternal LV short axis were captured for LV torsion. Images were standardized at 3 cardiac cycles with the frame rate of $60-80 \mathrm{~Hz}$. Basal and apical rotation of the 6 segments from each view were analyzed and the final torsion generated using Echopac version 113, reversion 1.2.

\subsection{Images Selection}

All prior images from our database were reviewed. Studies that did not fulfill the above criteria were excluded from the study. Refer to figure 1 for details.

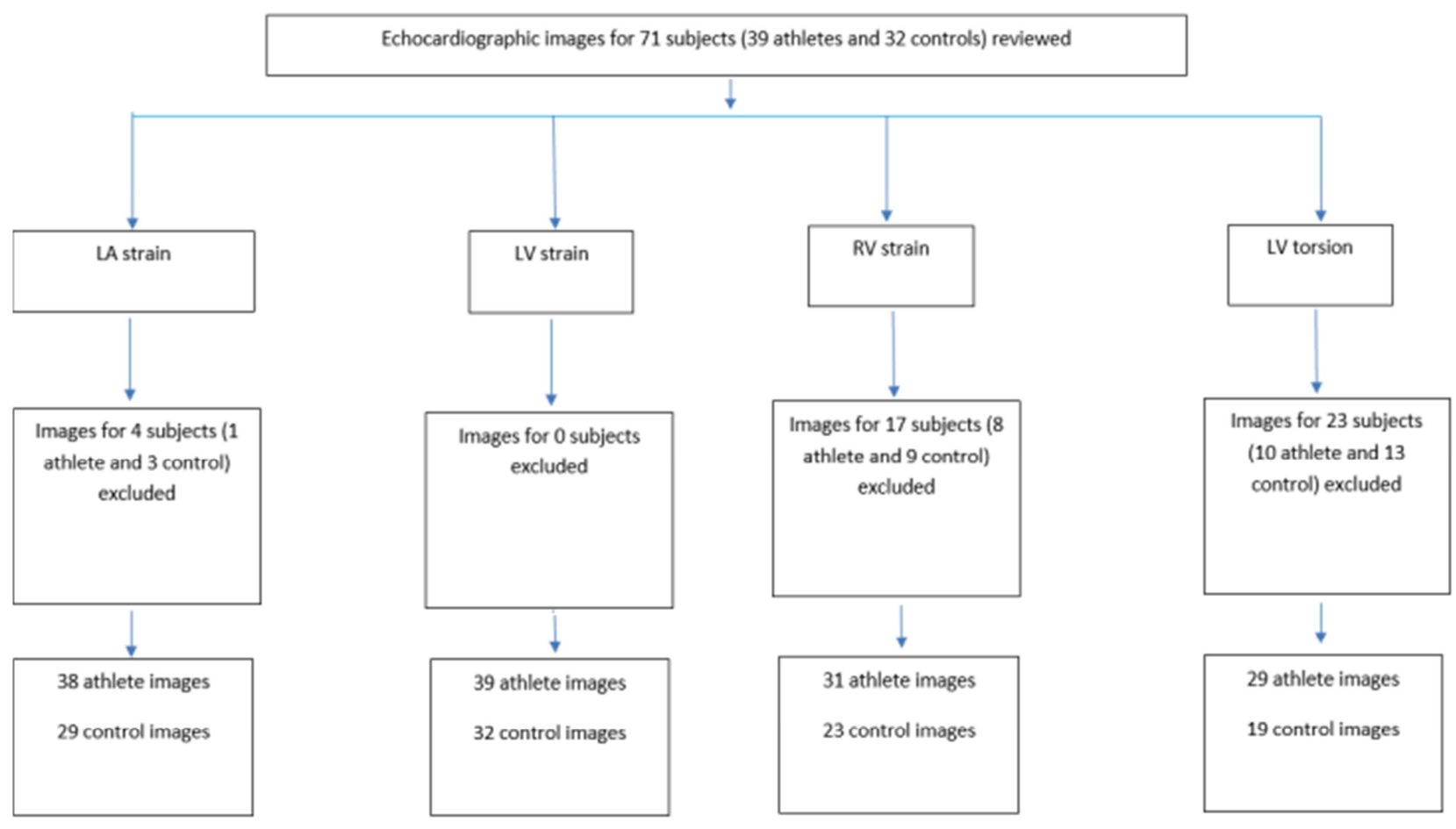

Figure 1. Workflow for processing echocardiographic images. 


\subsection{Statistical Analysis}

The patient's baseline characteristics were analyzed using chi square and independent $t$ tests. The nonparametric echocardiographic data was mainly analyzed using a MannWhitney test with a probability value $<0.05$ was considered significant. All statistical analysis was performed using SPSS version 25 (IBM Corp, New York, USA).

\section{Results}

A total of 71 subjects, 39 athletes and 32 control subjects were recruited. All participants were of Asian descent. One athlete was noted to have an atrial septal defect and was subsequently excluded from the study. Other participants had no significant cardiac pathologies.

Table 1. Characteristics of study participants at baseline.

\begin{tabular}{llll}
\hline & Control & Athletes & p value \\
\hline Gender & & & \\
Male & $11 / 17(65 \%)$ & $29 / 33(88 \%)$ & 0.024 \\
Age (mean \pm SD) years & $28.8 \pm 4.6$ & $36.4 \pm 4.7$ & 0.07 \\
Height (mean \pm SD) meters & $1.66 \pm 0.08$ & $1.69 \pm 0.08$ & 0.284 \\
Weight (mean \pm SD) k & $60.4 \pm 0.08$ & $69 \pm 0.08$ & 0.234 \\
Resting blood pressure & & & \\
Systolic (mean \pm SD) & $119.3 \pm 14.8$ & $120.3 \pm 12.7$ & 0.936 \\
$\begin{array}{l}\text { Diastolic (mean } \pm \text { SD) } \\
\text { Resting heart rate (mean } \pm\end{array}$ & $71.2 \pm 9.3$ & $70.7 \pm 8.9$ & 0.904 \\
SD) & $63.6 \pm 8.7$ & $66.5 \pm 10.2$ & 0.352 \\
\hline
\end{tabular}

Baseline characteristics

The baseline characteristics are summarized in table 1 and were discussed in our previous publication. [7] The athletes group consisted of mainly male (88\%) and endurance (94\%) athletes.
Table 2. Athlete subgroup based on event.

\begin{tabular}{ll}
\hline Type of Sport & N (\%) \\
\hline Triathlon athletes & $15(45 \%)$ \\
Marathon runner & $7(21.2 \%)$ \\
Mountaineer & $3(9.0 \%)$ \\
Body builder & $2(6.0 \%)$ \\
Boxer & $2(6.0 \%)$ \\
Squash & $2(6.0 \%)$ \\
Sprinter & $1(3.0 \%)$ \\
Sailing & $1(3.0 \%)$ \\
\hline
\end{tabular}

Echocardiographic results

The echocardiographic parameters are summarized in table 3.

The LV strain of the athletes group was lower as compared to the control group $(-19.0 \pm 2.0$ vs $-20.3 \pm 1.8$, $\mathrm{p}=0.011$ ), which was statistically significant. Furthermore, both LV torsion $(14.3 \pm 17.8 \%$ s $14.4 \pm 6.7 \%$ s, $\mathrm{p}=0.031)$ and Lateral $\mathrm{S}^{\prime}(7.21 \pm 1.4$ vs $8.7 \pm 1.6, \mathrm{p}=0.001)$ showed small but statistically significant decreases in the athletes group versus the controls.

There was no significant difference in terms of the doppler measured indices of Diastology (E/E', E/A Septal and Lateral E') between the athletes and controls.

The athletes group demonstrated a significantly lower Basal RV free wall strain as compared to the controls ($19.8 \pm 5.5$ vs $-26.5 \pm 6.4 \mathrm{P}<0.001$ ). The mid RV strain was marginally higher in the athletes group versus the controls ($25.0 \pm 4.3$ vs $-24.7 \pm 15.3 \mathrm{P}=0.023)$. There were however no significant differences in average GLS of the RV when comparing both groups $(-23.5 \pm 10.9$ vs $-24.0 \pm 11.1, \mathrm{p}=0.224)$.

There were also no significant differences in LA strain (37.4 \pm 6.7 vs $38.8 \pm 8.1, \mathrm{p}=0.44)$ and TDI Septal $\mathrm{A}^{\prime}$ and Lateral A' between the two groups.

Table 3. Echocardiographic parameters of study participants.

\begin{tabular}{llll}
\hline Parameters & Control $($ mean \pm SD) & Athletes $($ mean \pm SD) & p value \\
\hline LV end diastolic diameter $(\mathrm{mm})$ & $46 \pm 4.5$ & $52 \pm 3.6$ & $<0.001$ \\
LV end diastolic volume $(\mathrm{mL})$ & $67 \pm 18.7$ & $89.7 \pm 21$ & $<0.001$ \\
LV end diastolic volume index $\left(\mathrm{mL} / \mathrm{m}^{2}\right)$ & $41.1 \pm 11.1$ & $51.3 \pm 8.8$ & 0.002 \\
LA volume $(\mathrm{mL})$ & $37.8 \pm 12.6$ & $59.7 \pm 14.8$ & $<0.001$ \\
LA volume index $\left(\mathrm{mL} / \mathrm{m}^{2}\right)$ & $23.4 \pm 6.7$ & $34.7 \pm 7.9$ & $<0.001$ \\
RV internal diameter $(\mathrm{mm})$ & $18 \pm 4.0$ & $25 \pm 4.8$ & $<0.001$ \\
LV mass $(\mathrm{g})$ & $110.5 \pm 35.2$ & $149.1 \pm 35.1$ & 0.001 \\
LV mass index $\left(\mathrm{g} / \mathrm{m}^{2}\right)$ & $67.7 \pm 17.0$ & $85.2 \pm 18.0$ & 0.002 \\
LV wall thickness $(\mathrm{mm})$ & $7 \pm 1.2$ & 0.025 \\
LV ejection fraction $(\%)$ & $66.5 \pm 6.5$ & $8 \pm 1.2$ & 0.246 \\
E/A & $1.9 \pm 0.5$ & $63.4 \pm 7.3$ & 0.139 \\
DT & $182.5 \pm 40.6$ & $2.08 \pm 0.65$ & 0.153 \\
RWT & $0.30 \pm 0.06$ & $168.3 \pm 43.8$ & 0.952 \\
IVRT & $87.3 \pm 13.3$ & $0.28 \pm 0.05$ & 0.084 \\
Septal S' & $7.29 \pm 1.2$ & $93.0 \pm 16.4$ & 0.668 \\
Septal E' & $10.6 \pm 1.9$ & $7.33 \pm 1.3$ & 0.601 \\
Septal A' & $7.0 \pm 1.2$ & $10.5 \pm 1.9$ & 0.823 \\
Lateral S' & $8.7 \pm 1.6$ & $7.2 \pm 2.1$ & 0.001 \\
Lateral E' & $12.1 \pm 1.8$ & $7.21 \pm 1.4$ & 0.535 \\
Lateral A' & $5.9 \pm 1.6$ & $12.2 \pm 2.3$ & 0.056 \\
Average E/E' & $7.47 \pm 1.4$ & $5.3 \pm 2.1$ & 0.489 \\
LA Strain & $38.8 \pm 8.1$ & $7.18 \pm 1.7$ & 0.44 \\
GLS & $-20.3 \pm 1.8$ & $37.4 \pm 6.7$ & 0.011 \\
RV strain & & $-19.0 \pm 2.0$ & \\
\hline
\end{tabular}




\begin{tabular}{clll}
\hline Parameters & Control (mean \pm SD) & Athletes (mean \pm SD) & p value \\
\hline Basal RV & $-26.5 \pm 6.4$ & $-19.8 \pm 5.5$ & 0.001 \\
Mid RV & $-24.7 \pm 15.6$ & $-25.0 \pm 4.3$ & 0.023 \\
Distal RV & $-29.7 \pm 5.4$ & $-29.8 \pm 5.2$ & 0.768 \\
Average & $-24.0 \pm 11.1$ & $-23.5 \pm 10.9$ & 0.224 \\
LV torsion $\%$ s) & $14.4 \pm 6.7$ & $14.3 \pm 17.8$ & 0.031 \\
\hline
\end{tabular}

\section{Discussion}

\subsection{Function}

Early systolic dysfunction of the LV is suggested in our study with athletes showing marginally lower GLS in comparison to controls, and no significant difference in LV ejection fraction.

2 studies, one in soccer players and another in endurance athletes showed that endurance athletes seem to demonstrate impaired apical circumferential LV strain. [8, 9] Majority of our cohort were endurance/ mixed athletes $(31 / 33,94 \%)$ with eccentric hypertrophy, who could have contributed to the impaired LV strain. Interestingly, impairment in LV strain seems to be reversible in endurance athletes after 10 years of deconditioning. [10]

\subsection{Torsion}

Impaired LV strain rates have been demonstrated to translate into impaired LV torsion. [9] LV torsion has been shown to deteriorate after triathletes undergo ultralong endurance exercise either due to a change in preloading conditions or alteration in the balance of transmural strain gradients. [11] Our findings of marginally impaired LV torsion $(14.3 \pm 17.8 \%$ s vs $14.4 \pm 6.7 \% \mathrm{~s}, \mathrm{p}=0.031)$ and Lateral $\mathrm{S}$ ' (7.21 \pm 1.4 vs $8.7 \pm 1.6, p=0.001)$ in our athletes correlated well with the findings of other studies. [9, 12]

\subsection{Diastology}

Our study did not describe any significant difference in LV diastology between the athletes and controls by Doppler Echocardiography. [12] In contrast, echocardiographic strain imaging and cardiac MRI have been used to quantify untwisting rates in athletes. Higher peak LV diastolic untwisting rates in endurance athletes as opposed to controls seem to suggest supernormal diastolic filling. [9, 13]

\subsection{LA Function}

A recent study comparing athletes in 'High Dynamic High Static' sports - boxers and triathletes to 'Low Dynamic High Static' Sports -- weightlifters with sedentary controls observed that only athletes participating in in 'High Dynamic High Static' experienced biatrial dilatation. [14] Our athletes (predominantly triathletes and marathon runners) had similar Left atrial dilatation compared to controls, but no change in LA strain, suggesting that the increase in LA compliance does not compromise LA contractility.

\section{5. $R$ V Function}

The impaired RV Strain at the RV basal free wall has been observed in similar cohorts of endurance athletes. [15] One view is that these changes represent an adaptation to $\mathrm{RV}$ dilatation and that these changes resolve at peak exercise, demonstrating contractile reserve. [16] However, another study by Gerche et al using both strain echocardiography and CMR demonstrated decreases in RV deformation immediately after endurance races. [17] They postulated that the RV, being less muscular than the LV, could be more susceptible to injury from the increase in preload during endurance exercise. Our findings of a marginally higher mid $\mathrm{RV}$ free wall strain could suggest a compensatory response to the impaired basal RV function in athletes.

\section{Limitations}

Our study had a number of limitations. As the echo images were retrieved from a database and post processed, there were several suboptimal images which had to be excluded from analysis. However, we were still able to analyze most of the images. Also, given the natures of recruitment of the original cohort, our results represent mostly endurance induced cardiac remodeling, due to inadequate representation of strength or mixed sports. We were also unable to comment on the temporal relationship between exercise exposure and exercise induced cardiac remodeling as this was a cross sectional study. We were not able to analyze twisting and untwisting of the LV, and also did not assess radial and circumferential strain of the LV, due to limitation of software and image acquisition.

\section{Conclusions}

Our findings of impaired LV strain and torsion as well basal RV free walls strain in the South East Asian athletes likely represent early changes of exercise induced cardiac remodelling. The higher mid RV free wall strain could represent compensatory response to the impaired basal RV function in athletes. These findings support the use of myocardial strain imaging as a modality to detect subclinical exercise induced cardiac remodeling.

\section{Conflict of Interest}

The authors declare no conflicts in interest.

\section{References}

[1] Baggish AL, Wood MJ. Athlete's heart and cardiovascular care of the athlete: scientific and clinical update. Circulation. 2011; 123 (23): 2723-35. 
[2] D'Andrea A, Bossone E, Radmilovic J, Caso P, Calabro R, Russo MG, et al. The role of new echocardiographic techniques in athlete's heart. F1000Res. 2015; 4: 289.

[3] Caso P, D'Andrea A, Galderisi M, Liccardo B, Severino S, De Simone L, et al. Pulsed Doppler tissue imaging in endurance athletes: relation between left ventricular preload and myocardial regional diastolic function. The American journal of cardiology. 2000; 85 (9): 1131-6.

[4] Tumuklu MM, Ildizli M, Ceyhan K, Cinar CS. Alterations in left ventricular structure and diastolic function in professional football players: assessment by tissue Doppler imaging and left ventricular flow propagation velocity. Echocardiography. 2007; 24 (2): 140-8.

[5] Lewis JF, Spirito P, Pelliccia A, Maron BJ. Usefulness of Doppler echocardiographic assessment of diastolic filling in distinguishing "athlete's heart" from hypertrophic cardiomyopathy. Br Heart J. 1992; 68 (3): 296-300.

[6] Richand V, Lafitte S, Reant P, Serri K, Lafitte M, Brette S, et al. An ultrasound speckle tracking (two-dimensional strain) analysis of myocardial deformation in professional soccer players compared with healthy subjects and hypertrophic cardiomyopathy. The American journal of cardiology. 2007; 100 (1): $128-32$.

[7] Keh YS, Tan PJ, Chai SC, Tan B, Tong KL. Physiologic Limits of Cardiac Remodelling in Asian Competitive Athletes - A Single Centre Study. Ann Acad Med Singapore. 2018; 47 (6): $230-2$.

[8] Santoro A, Alvino F, Antonelli G, Caputo M, Padeletti M, Lisi $\mathrm{M}$, et al. Endurance and Strength Athlete's Heart: Analysis of Myocardial Deformation by Speckle Tracking Echocardiography. J Cardiovasc Ultrasound. 2014; 22 (4): 196-204.

[9] von Lueder TG, Hodt A, Gjerdalen GF, Steine K. Left ventricular biomechanics in professional football players. Scand J Med Sci Sports. 2018; 28 (1): 187-95.
[10] Demirelli S, Sam CT, Ermis E, Degirmenci H, Sen I, Arisoy A, et al. Long-Term Cardiac Remodeling in Elite Athletes: Assessment by Tissue Doppler and Speckle Tracking Echocardiography. Echocardiography. 2015; 32 (9): 1367-73.

[11] Nottin S, Doucende G, Schuster I, Tanguy S, Dauzat M, Obert P. Alteration in left ventricular strains and torsional mechanics after ultralong duration exercise in athletes. Circulation Cardiovascular imaging. 2009; 2 (4): 323-30.

[12] Simsek Z, Hakan Tas M, Degirmenci H, Gokhan Yazici A, Ipek E, Duman H, et al. Speckle tracking echocardiographic analysis of left ventricular systolic and diastolic functions of young elite athletes with eccentric and concentric type of cardiac remodeling. Echocardiography. 2013; 30 (10): 1202-8.

[13] Esch BT, Scott JM, Haykowsky MJ, Paterson I, Warburton DE, Cheng-Baron J, et al. Changes in ventricular twist and untwisting with orthostatic stress: endurance athletes versus normally active individuals. J Appl Physiol (1985). 2010; 108 (5): 1259-66.

[14] McClean G, George K, Lord R, Utomi V, Jones N, Somauroo $\mathrm{J}$, et al. Chronic adaptation of atrial structure and function in elite male athletes. European heart journal cardiovascular Imaging. 2015; 16 (4): 417-22.

[15] D'Andrea A, La Gerche A, Golia E, Teske AJ, Bossone E, Russo MG, et al. Right heart structural and functional remodeling in athletes. Echocardiography. 2015; 32 Suppl 1: S11-22.

[16] La Gerche A, Burns AT, D'Hooge J, Macisaac AI, Heidbuchel H, Prior DL. Exercise strain rate imaging demonstrates normal right ventricular contractile reserve and clarifies ambiguous resting measures in endurance athletes. Journal of the American Society of Echocardiography: official publication of the American Society of Echocardiography. 2012; 25 (3): 253-62 e1.

[17] La Gerche A, Burns AT, Mooney DJ, Inder WJ, Taylor AJ, Bogaert J, et al. Exercise-induced right ventricular dysfunction and structural remodelling in endurance athletes. European heart journal. 2012; 33 (8): 998-1006. 